\title{
A narrative review of diagnosis of infective endocarditis-imaging methods and comparison
}

\author{
Sanjeet Singh Avtaar Singh ${ }^{1,2}$, Marco Fabio Costantino ${ }^{3}$, Gianpaolo D'Addeo ${ }^{3}$, Damiano Cardinale ${ }^{3}$, \\ Rosario Fiorilli ${ }^{3}$, Francesco Nappi ${ }^{4}$ \\ ${ }^{1}$ Department of Cardiac Surgery, Golden Jubilee National Hospital. Glasgow, UK; ${ }^{2}$ Institute of Cardiovascular and Medical Sciences, University of \\ Glasgow, Glasgow, UK; ${ }^{3}$ Cardiovascular Department, Institute of Cardiology, S. Carlo Hospital, Basilicata, Italy; ${ }^{4}$ Department of Imaging, Centre \\ Cardiologique du Nord de Saint-Denis, Paris, France \\ Contributions: (I) Conception and design: MF Costantino, G D’Addeo; (II) Administrative support: None; (III) Provision of study materials or \\ patients: None; (IV) Collection and assembly of data: MF Costantino, D Cardinale, SS Avtaar Singh; (V) Data analysis and interpretation: MF \\ Costantino, G D’Addeo, D Cardinale, SS Avtaar Singh; (VI) Manuscript writing: All authors; (VII) Final approval of manuscript: All authors. \\ Correspondence to: Francesco Nappi, MD. Department of Cardiac Surgery, Centre Cardiologique du Nord, 36 Rue des Moulins Gémeaux, 93200 \\ Saint-Denis, Paris, France. Email: francesconappi2@gmail.com.
}

\begin{abstract}
The profile of infective endocarditis (IE) has changed over the past few decades. The modified Duke's criteria is currently employed for diagnosis of IE. Emphasis on imaging modalities however, have been increasing due to the variety of presenting symptoms leading to diagnostic conundrums. This wide range of diagnostic tools must be adapted to permit localization of the infectious field which may involve multiple valves on either side of the heart. The availability of such diagnostic tools is also variable in different centres. The use of echocardiography has long been the default position, however the lack of specificity and sensitivity especially in prosthetic valve endocarditis has been highlighted throughout the literature. We therefore aimed to look at the different imaging modalities available and the strengths and weaknesses of each of these modalities to enhance the diagnostic yield and allow timely intervention for this condition. We highlight the role of the different forms of echocardiography, multi-detector computed tomography (MDCT), Nuclear Medicine, Magnetic Resonance Imaging and identify the special indications such as right sided infective endocarditis (RSIE) and cardiac implantable electronic device (CIED) endocarditis. Input from a specialist heart team is essential to ensure timely diagnosis and care are afforded. The role of alternative imaging techniques such as nuclear medicine in determining timing of cardiac surgery should be evaluated further by randomised trials.
\end{abstract}

Keywords: Infective endocarditis (IE); multi-detector computed tomography (MDCT); transthoracic echocardiography (TTE); transoesophageal echocardiography (TEE); magnetic nuclear resonance (MRI); nuclear medicine

Submitted Jun 07, 2020. Accepted for publication Jul 10, 2020.

doi: 10.21037/atm-20-4555

View this article at: http://dx.doi.org/10.21037/atm-20-4555

\section{Introduction}

Cardiac imaging and positive blood cultures constitute the major criteria for the diagnosis of infective endocarditis (IE) according to the Modified Duke Criteria (1). In the 21st century, IE has a prevalence of 3-10 per 100,000 people which starkly rises to 14.5 per 100,000 if aged $>70$ years old (2). However, blood cultures can be negative in up to $30 \%$ of confirmed IE, placing greater emphasis on the role of cardiac imaging. There is a large spectrum of imaging for IE encompassing anatomical features (multi-detector computed tomography, MDCT), anatomo-functional findings (echodoppler), and hybrid approaches combining anatomical and metabolic features such as nuclear medicine modalities. This wide range of diagnostic tools must be adapted to the 
Table 1 Sensitivities and specificities of echocardiography for native and prosthetic valves

\begin{tabular}{lcccc}
\hline & \multicolumn{2}{c}{ Vegetations on native valve } & \multicolumn{2}{c}{ Vegetation on prosthetic valve } \\
\cline { 2 - 5 } & TTE & TEE & TTE & TEE \\
\hline Sensitivity\% & $25-87$ & $87-100$ & $22-65$ & $89-99$ \\
Specificity\% & $79-96$ & $91-100$ & $48-98$ & $87-100$ \\
\hline
\end{tabular}

TTE, transthoracic echocardiography; TEE, transoesophageal echocardiography.

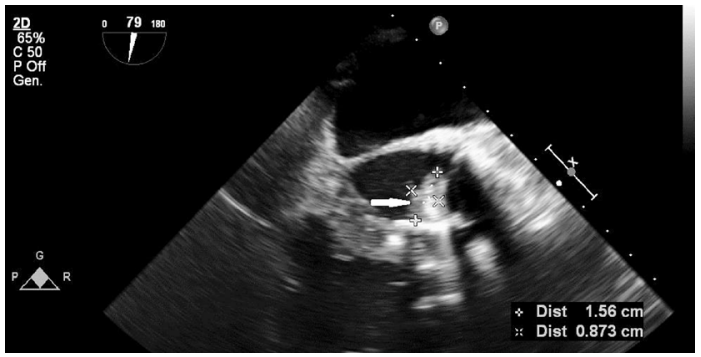

Figure 1 Large vegetation on bioprosthetic mitral valve (2D transoesophageal echocardiography).

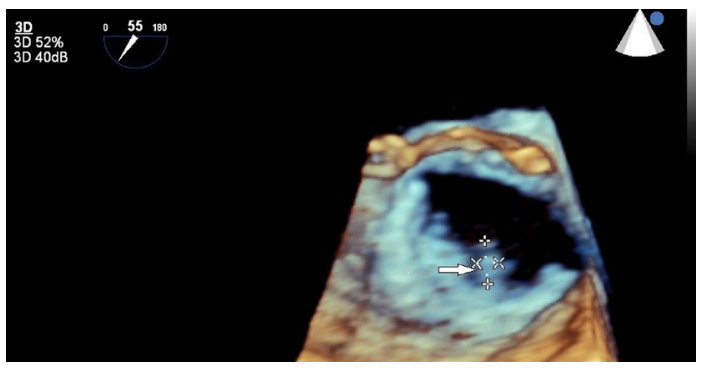

Figure 2 Large vegetation on bioprosthetic mitral valve (3D transoesophageal echocardiography).

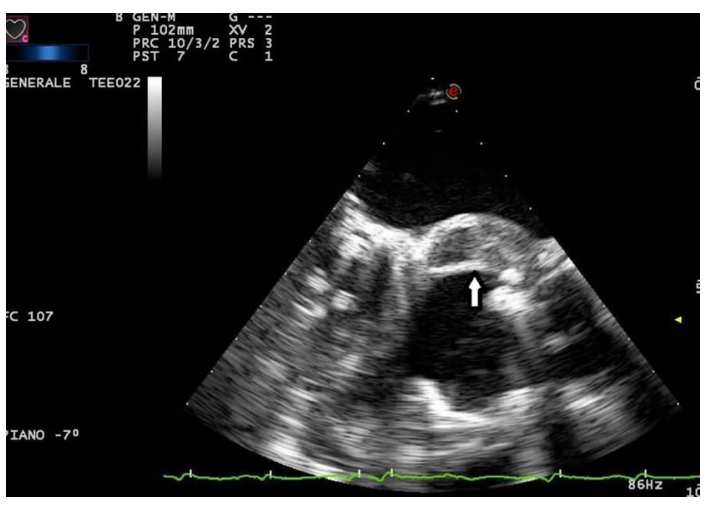

Figure 3 Large abscess on mitroaortic curtain (2D transoesophageal echocardiography). variability of the clinical presentations of IE, manifesting itself as acute or subacute alongside localization of the infectious field which may involve multiple valves on either side of the heart. IE can manifest itself either as a pathology of the native valve, prosthetic valve, or on implantable cardiac devices. It may also coexist with other comorbidities such as chronic kidney disease or cerebrovascular impairment.

The aim of this article is to compare the different imaging modalities for the diagnosis of IE.

We reviewed the literature for the current guidelines on imaging from ESC and ACC/AHA alongside relevant studies from 2009 to 2019.

We present the review in accordance with the Narrative Review reporting checklist (available at http://dx.doi. org/10.21037/atm-20-4555).

\section{Outlook on diagnostic tools}

\section{Echocardiography}

Both transthoracic echocardiography (TTE) and transoesophageal echocardiography (TEE) are the main imaging modalities for diagnosis of IE The European Society of Cardiology (ESC) classify echocardiography as $1 \mathrm{~B}$ recommendation with echocardiographic results an important Duke criterion (2). Diagnosis based on echocardiographic images has high sensitivity and specificity to detect vegetations, a distinctive sign of IE, particularly if it measures $>5 \mathrm{~mm}$ (Table 1) and for valve detachment (3). It is however less accurate when it comes to detecting abscesses, aneurysms, pseudoaneurysms, and fistulas. Echocardiography allows the rapid detection of vegetations (Figures 1,2), by measuring the major and minor axes of these, in addition to allowing differentiation from other intracardiac masses, informing the operator on location, mobility (fixed, mobile, oscillating) (Figure 3), echogenicity and potential complications such as the presence of leaks, 
Table 2 Sensitivity and specificity of echocardiography in detecting abscesses

\begin{tabular}{lccccc}
\hline & \multicolumn{3}{c}{ Abscesses } & \multicolumn{2}{c}{ Periprosthetic abscesses } \\
\cline { 2 - 5 } & TTE & TEE & TTE & TEE \\
\hline Sensitivity\% & $28-36$ & $80-100$ & - & $48-87$ \\
Specificity\% & 99 & 95 & - & $95-99$ \\
\hline
\end{tabular}

TTE, transthoracic echocardiography; TEE, transoesophageal echocardiography.

Table 3 Differentiating vegetations from other masses

\begin{tabular}{ll}
\hline Vegetation & Other Masses \\
\hline Attached to valve, upstream side & Attached to valve downstream side \\
Mobile, oscillating & Nonmobile \\
Irregular shape & Smooth surface o fibrillar \\
Low reflectance & High echogenicity \\
Associated valvular regurgitation o perivalvular lesions & Absence of valvular involvement \\
High risk of embolization (if size $>1 \mathrm{~cm})$ & \\
\hline
\end{tabular}

fistulas, and dehiscences $(2,4)$.

Echocardiography, however, does have several limitations. It is heavily operator dependent and is subject to interoperator variability. In addition, TTE is dependent on having satisfactory imaging windows which are often patient dependent with some having poorly echogenic chests. It is less sensitive for smaller vegetations measuring $<5 \mathrm{~mm}$ (4). In the elderly, bulky calcific deposits on structures may also make image interpretation difficult. In patients with prosthetic valves, acoustic shadowing by the metallic component of prosthetic valves (on Left Ventricular Outflow Tract in aortic and on Left Atrium in mitral prostheses) may also contribute to its lower sensitivity and specificity $(4,5)$. Some of these patients may also have a submammary prosthesis, which may affect the imaging windows, thereby reducing the sensitivity and specificity of this modality. Echocardiography also is of limited value in evaluating extracardiac lesions and may sometimes lead to delays in detecting intracardiac lesions. The diagnosis of abscesses (Figure 3) can be challenging for echocardiographers with lower sensitivity and specificity (Tables 2,3).

\section{Multidetector computed tomography}

MDCT is able to examine cardiac structures and coronary arteries due to technological innovations enabling images at $0.5 \mathrm{~mm}$ slices.
Both TTE and TEE have a lower sensitivity in detecting abscesses in the presence of prosthetic valve(s) and electrophysiological devices (Table 2,3) $(3,6)$.

In 115 patients evaluated using Duke's criteria for IE, TEE had a sensitivity of detecting abscesses of $48 \%$, whereas $64 \%$ missed detecting abscesses that were located near bulky calcific deposits on the posterior mitral valve leaflet (5). MDCT has increased spatial resolution and unaffected by acoustic shadowing. MDCT can evaluate coronary artery anatomy (in the setting of ischemic disease or congenital defects). The enhanced spatial resolution permits the evaluation of perivalvular lesions (abscesses, fistulas, pseudoaneurysms, and extension into the cardiac skeleton) (Figure 4). MDCT can also detect primary or IE related pneumonic diseases as abscesses or necrotic deposits. It facilitates effective imaging of the tricuspid annulus and leaflets (Table 4) (7).

As the tricuspid valve is anteriorly located with a $45^{\circ}$ rotation, imaging it is often challenging using TEE with mid-oesophageal and transgastric views (8).

TTE imaging is often inadequate because the tricuspid leaflet is thinner than the mitral and while its annulus is saddle-shaped (with the anteroseptal and posterolateral axes on the top of the saddle (Table 5) (9).

\section{Magnetic nuclear resonance (MRI)}

There are anecdotal cases of early diagnosis of IE using 
MRI rather than echocardiography. Cerebral MRI is the most sensitive technique to detect cerebral emboli (10). It should be done in the presence of neurological symptoms whereas its use is controversial in asymptomatic patients. However, it is often performed in asymptomatic patients who are due to undergo cardiac surgery.

\section{Nuclear medicine}

PET-CT can identify areas of increased glucose metabolism corresponding to active inflammation which can be mapped

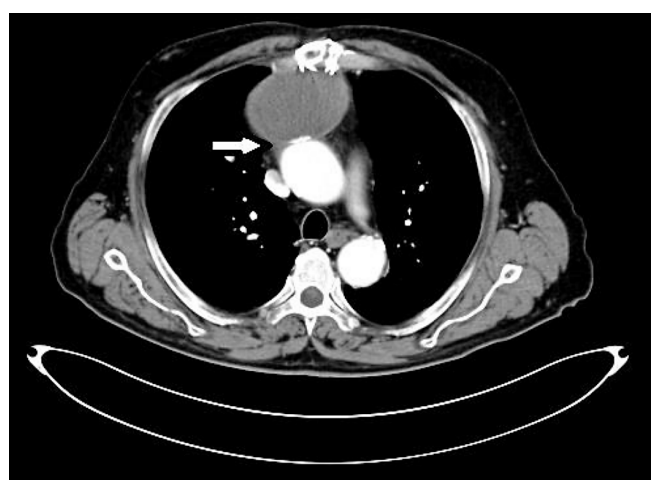

Figure 4 Huge periaortic pseudoaneurysm. by conventional CT to provide anatomical landmarks $(2,11)$. PET-CT utilizes white blood cells (WBC) of the suspected patient, tagged in vitro with Technetium-99, and then reinjected into the same patient. Tagged WBC migrates to the infected site. The relative radioactivity is recorded by a Gamma chamber (SPECT) and then by a hybrid Gamma Chamber (SPECT-CT) (12). It is necessary to wait for 24 hours and we have high specificity and less sensitivity. Again with WBC tagged 'in vivo' with F18fluorodeoxyglucose (FDG) we wait for 1.5-2 hours and we have high sensitivity and less specificity. The biggest advantages offered by this modality include the ability to detect early infective disease processes from inconclusive imaging from other modalities (3D Echocardiography/ MDCT). It enables the evaluation of extracardiac structures such as cutaneous pouches (13) or ventricular assist device (VAD) drivelines (14). In addition, it is able to detect sources of emboli that were possibly missed.

Despite the benefits of PET imaging, there are several pitfalls to be mindful of. These include the high falsepositive rate if performed early post-implantation of a prosthetic valve (15). Also, due to its dependence on FDG uptake, it may have a false negative in several instances such as low inflammatory activity, microorganisms with slow growth or biofilm, or prolonged antibiotic therapy $(15,16)$.

Table 4 Sensitivity and Specificity of MDCT for detecting IE changes

\begin{tabular}{lcccc}
\hline & Vegetations & Abscesses & Pseudoaneurysm & Leaflet/cusp perforations \\
\hline Sensitivity MDCT & $96 \%$ & $97 \%$ & $97 \%$ & $<50 \%$ \\
Specificity MDCT & $99 \%$ & $75 \%$ & $75 \%$ & $89 \%$ \\
\hline
\end{tabular}

MDCT, multi-detector computed tomography; IE, infective endocarditis.

Table 5 Differences between Echocardiography and MDCT in detecting IE changes

\begin{tabular}{ll}
\hline Echocardiography & MDCT \\
\hline High spatial resolution & High spatial resolution \\
High sensitivity and specificity & Able to detect perivalvular lesion (abscess-pseudoaneurysm) \\
Permits evaluation of Jet, shunt and transvalvular gradient & Permits to evaluate annular and leaflet bulky calcium \\
Bedside & Able to evaluate aortic root and coronary arteries \\
Able to detect perforation of leaflet or cusps & Allows tricuspidalic anulus and leaflet imaging \\
Permits to plan cardiac surgery & Evaluate extracardiac structures \\
Allows percutaneous reparation of leaks (Figure 5) & \\
Without RX ray or contrast agent & \\
\hline
\end{tabular}

MDCT, multi-detector computed tomography; IE, infective endocarditis. 


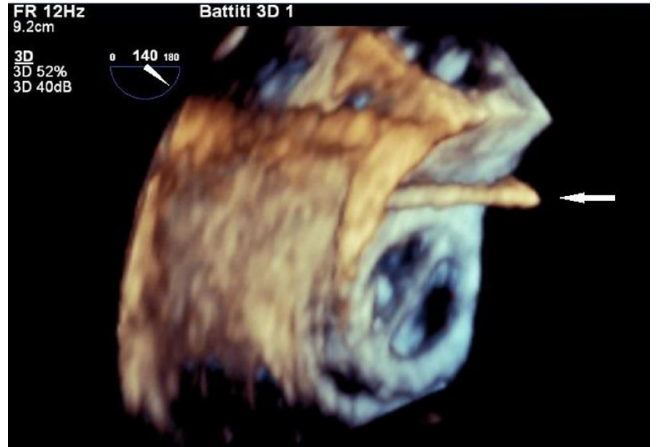

Figure 5 Percutaneous closure of mitral prosthetic leak (3D transoesophageal echocardiography).

\section{Specific pathoanatomic lesions}

\section{Trigone abscesses}

One such complication of PVE is an abscess of the aortic root with periprosthetic leak with via the non-coronary sinus which may perforate the fibrous trigone causing a fistula in the left or right atrium. This lesion may directly affect the mitral annulus by detaching it through the central fibrous body to the septal tricuspid valve leaflet (17). These abscesses are often detected with RT3D Echocardiography with MDCT providing additional information.

\section{Right-sided infective endocarditis (RISIE)}

Compared to left-sided IE (LISIE), RISIE usually presents with larger vegetations possibly due to a delay in diagnosis $(18,19)$. There has also been a shift in the epidemiology of the disease with more Staphylococcus aureus infections probably due to the increased proportion of active Intravenous Drug Users (IVDU) and implantable cardiac devices who present (19). In one study, the median vegetation size in RISIE is $2.2(1.8-2.9) \mathrm{cm}$ compared to $1.5(1.0-2.0) \mathrm{cm}$ in LISIE (20). Complications of RISIE are usually related to pulmonary embolic events, whereas abscesses, perforation, and fistulas are more prevalent in LISIE (19).

TTE is the first-line diagnostic tool with TEE required only in a minority of cases (when results of TTE is inconclusive in presence of prosthetic valve or intracardiac device) due to the anterior position of the tricuspid valve (21).

In a study of suspected right-sided endocarditis, TTE performed as well as TEE in the detection of vegetations (22). RT3D TEE also has a role in patients with tricuspid valve IE as it allows enhanced visualization of the tricuspid valve apparatus, vegetations, and surrounding structures compared to 2D TEE (23). In patients with a tricuspid ring or tricuspid valve prosthesis and/or electrophysiological devices, RT3D echocardiography can detect vegetations and their exact origins, permitting real-time therapeutic interventions or device extraction (Figure 5).

\section{Cardiac implantable electronic device (CIED)}

The presence of electrophysiological leads is associated with IE in $<2 \%$ of patients (24) although the rate of infections after primary device implantation was reported to be 4.82/1,000 device days in a large Danish study (25). TTE has poor sensitivity and specificity compared to both TEE and RT 3D. The challenge with such modalities however is in discerning and differentiating the other structures and masses amidst artefact (Table 1). Nuclear medicine scans such as 18-FDG PET do provide a viable and useful alternative (26). Sarrazin et al. noted that positive PET/CT results correlated well with clinical findings, microbiologic data, and echocardiographic evidence of device infection (27). In their study, patients with negative PET/CT were managed conservatively with antimicrobial therapy alone, without device extraction (27). For pockets of infections, FDG activity analysis notably yielded a very high diagnostic accuracy.

\section{Discussion}

In some instances, multimodality imaging may be required to facilitate early diagnosis of IE to afford timely therapy to patients either by antibiotic or cardiac surgery. The primary indications for early cardiac surgery are heart failure, uncontrolled infection and prevention of embolization (2). Early surgery is indicated during initial hospitalization in AHA-ACC Guidelines (28) whereas the European guidelines have set a more rigorous target of emergent surgery (within 24 hours) (2).

Echocardiography is the primary imaging modality in the instance of heart failure as it allows evaluation of right and left ventricle function and quantitative evaluation of valvular function (28). Vegetations measuring $>15 \mathrm{~mm}$ by echocardiography should be highlighted due to their risk of imminent embolization (29). Anteriorly located structures such as the tricuspid valve may be best evaluated using TTE.

Posteriorly located structures however, are best imaged using TEE. RT3D echocardiography may be useful in 
Table 6 The specific advantages and pitfalls of diagnostic imaging methods

\begin{tabular}{|c|c|c|c|c|}
\hline & Echo TTE/ETT & MDCT & CMR & Nuclear medicine \\
\hline Evaluation Jets, shunts & **** & No & $* * * *$ & No \\
\hline Evaluation of cardiac performance & 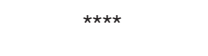 & $\star \star$ & $* \star \star *$ & No \\
\hline Early diagnosis & * & * & * & $* * *$ \\
\hline Extracardiac lesions & No & No & $* * *$ & **** \\
\hline Shadowing & $\star \star \star ~$ & * & 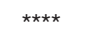 & No \\
\hline RSIE & $* * * *$ & $\star *$ & * & * \\
\hline Imaging of perivalvular lesions & $\star \star \star \star ~$ & $\star \star \star * *$ & * & * \\
\hline
\end{tabular}

TTE, transthoracic echocardiography; TEE, transoesophageal echocardiography; MDCT, multi-detector computed tomography; CMR, cardiac magnetic resonance; RSIE, right sided infective endocarditis.

detecting dynamic valve function and evaluating paravalvular leaks or honing in on the nature of the vegetations. For systemic uncontrolled infections, MDCT is more sensitive at detecting sequalae of disease such as abscess formation, pseudoaneurysm and fistulas and alongside extension to the cardiac fibrous skeleton due to the better spatial resolution (2). In addition, the metabolic activity of septic emboli is best evaluated using nuclear medicine imaging modalities. It helps with detection of potential infective sites even before direct imaging of anatomic lesions are possible which may expediate the diagnosis of IE. Cerebral MRI on the other hand is a useful tool to evaluate previous cerebral embolization. Previous embolization in presence of vegetations measuring $>10 \mathrm{~mm}$ is also gurean indication for surgery.

The specific advantages and pitfalls of diagnostic methods are summarized in Table 6 . The following is a stepwise guide to escalation of imaging.

(I) All patients with IE should receive a TTE and TEE to detect valvular lesions and perivalvular complications.

(II) MDCT should be used to evaluate the extension of perivalvular lesions when echocardiography images are suboptimal or inconclusive.

(III) Cerebral MRI should be used to evaluate neurologically symptomatic patients.

(IV) Nuclear medicine should be considered should there be diagnostic uncertainty or in the presence of CIED or PVE.

\section{Conclusions}

Diagnostic imaging of IE often requires a multimodal imaging approach with the choice of the various imaging techniques depending on the specific type of IE and location of the disease, the severity of the clinical status, appropriateness of contrast imaging and the modalities available. It requires input from a specialist heart team to ensure timely diagnosis and care are afforded. The role of alternative imaging techniques such as nuclear medicine in determining timing of cardiac surgery should be evaluated further by randomised trials.

\section{Acknowledgments}

Funding: None.

\section{Footnote}

Provenance and Peer Review: This article was commissioned by the editorial office, Annals of Translational Medicine for the series "Infective Endocarditis in the 21st Century". The article was sent for external peer review organized by the Guest Editor and the editorial office.

Reporting Checklist: The authors have completed the Narrative Review reporting checklist. Available at http:// dx.doi.org/10.21037/atm-20-4555 
Conflicts of Interest: All authors have completed the ICMJE uniform disclosure form (available at http:// dx.doi.org/10.21037/atm-20-4555). The series "Infective Endocarditis in the 21st Century" was commissioned by the editorial office without any funding or sponsorship. FN served as the unpaid Guest Editor of the series and serves as an unpaid editorial board member of Annals of Translational Medicine from Feb 2019 to Jan 2021. The authors have no other conflicts of interest to declare.

Ethical Statement: The authors are accountable for all aspects of the work in ensuring that questions related to the accuracy or integrity of any part of the work are appropriately investigated and resolved.

Open Access Statement: This is an Open Access article distributed in accordance with the Creative Commons Attribution-NonCommercial-NoDerivs 4.0 International License (CC BY-NC-ND 4.0), which permits the noncommercial replication and distribution of the article with the strict proviso that no changes or edits are made and the original work is properly cited (including links to both the formal publication through the relevant DOI and the license). See: https://creativecommons.org/licenses/by-nc-nd/4.0/.

\section{References}

1. Lang RM, Goldstein SA, Kronzon I, et al. ASE's comprehensive echocardiography. 2nd edition. Elselvier, 2015.

2. Habib G, Lancellotti P, Antunes MJ, et al. 2015 ESC Guidelines for the management of infective endocarditis: The Task Force for the Management of Infective Endocarditis of the European Society of Cardiology (ESC) Endorsed by: European Association for Cardio-Thoracic Surgery (EACTS), the European Association of Nuclear Medicine (EANM). Eur Heart J 2015;36:3075-128.

3. Salaun E, Habib G. Beyond Standard Echocardiography in Infective Endocarditis. Circ Cardiovasc Imaging 2018;11:e007626.

4. Habib G, Badano L, Tribouilloy C, et al. Recommendations for the practice of echocardiography in infective endocarditis. Eur J Echocardiogr 2010;11:202-19.

5. Hill EE, Herijgers $P$, Claus $P$, et al. Abscess in infective endocarditis: the value of transesophageal echocardiography and outcome: a 5-year study. Am Heart J 2007;154:923-8.

6. Feuchtner GM, Stolzmann P, Dichtl W, et al. Multislice computed tomography in infective endocarditis: comparison with transesophageal echocardiography and intraoperative findings. J Am Coll Cardiol 2009;53:436-44.

7. Erba PA, Pizzi MN, Roque A, et al. Multimodality Imaging in Infective Endocarditis. Circulation 2019;140:1753-65.

8. Bhatia A. Transesophageal echocardiography evaluation of tricuspid and pulmonic valves. Ann Card Anaesth 2016;19:S21-5.

9. Khalique OK, Cavalcante JL, Shah D, et al. Multimodality Imaging of the Tricuspid Valve and Right Heart Anatomy. JACC Cardiovasc Imaging 2019;12:516-31.

10. Champey J, Pavese P, Bouvaist H, et al. Value of brain MRI in infective endocarditis: a narrative literature review. Eur J Clin Microbiol Infect Dis 2016;35:159-68.

11. Chen W, Sajadi MM, Dilsizian V. Merits of FDG PET/ $\mathrm{CT}$ and Functional Molecular Imaging Over Anatomic Imaging With Echocardiography and CT Angiography for the Diagnosis of Cardiac Device Infections. JACC Cardiovasc Imaging 2018;11:1679-91.

12. Erba PA, Israel O. SPECT/CT in infection and inflammation. Clin Transl Imaging 2014;2:519-35.

13. Ahmed FZ, Arumugam P. 18F-FDG PET/CT now endorsed by guidelines across all types of CIED infection: Evidence limited but growing. J Nucl Cardiol 2019;26:971-4.

14. Akin S, Muslem R, Constantinescu AA, et al. 18F-FDG PET/CT in the Diagnosis and Management of Continuous Flow Left Ventricular Assist Device Infections: A Case Series and Review of the Literature. ASAIO J 2018;64:e11-9.

15. Swart LE, Gomes A, Scholtens AM, et al. Improving the Diagnostic Performance of (18)F-Fluorodeoxyglucose Positron-Emission Tomography/Computed Tomography in Prosthetic Heart Valve Endocarditis. Circulation 2018;138:1412-27.

16. Mahmood M, Kendi AT, Ajmal S, et al. Meta-analysis of 18F-FDG PET/CT in the diagnosis of infective endocarditis. J Nucl Cardiol 2019;26:922-35.

17. Akay MH, Danch MA, Cohn WE, et al. Reconstruction of the fibrous trigone. Tex Heart Inst J 2009;36:475-6.

18. Weber C, Gassa A, Eghbalzadeh K, et al. Characteristics and outcomes of patients with right-sided endocarditis undergoing cardiac surgery. Ann Cardiothorac Surg 2019;8:645-53.

19. Holland TL, Baddour LM, Bayer AS, et al. Infective endocarditis. Nat Rev Dis Primers 2016;2:16059-.

20. Leitman M, Dreznik Y, Tyomkin V, et al. Vegetation size in patients with infective endocarditis. Eur Heart $\mathrm{J}$ Cardiovasc Imaging 2012;13:330-8. 
21. San Román JA, Vilacosta I, López J, et al. Role of transthoracic and transesophageal echocardiography in right-sided endocarditis: one echocardiographic modality does not fit all. J Am Soc Echocardiogr 2012;25:807-14.

22. San Román JA, Vilacosta I, Zamorano JL, et al. Transesophageal echocardiography in right-sided endocarditis. J Am Coll Cardiol 1993;21:1226-30.

23. Naqvi TZ, Rafie R, Ghalichi M. Real-time 3D TEE for the diagnosis of right-sided endocarditis in patients with prosthetic devices. JACC Cardiovasc Imaging 2010;3:325-7.

24. Sandoe JAT, Barlow G, Chambers JB, et al. Guidelines for the diagnosis, prevention and management of implantable cardiac electronic device infection. Report of a joint Working Party project on behalf of the British Society for Antimicrobial Chemotherapy (BSAC, host organization), British Heart Rhythm Society (BHRS), British Cardiovascular Society (BCS), British Heart Valve Society (BHVS) and British Society for Echocardiography (BSE). J Antimicrob Chemother 2015;70:325-59.

25. Johansen JB, Jørgensen OD, Møller M, et al. Infection

Cite this article as: Avtaar Singh SS, Costantino MF, D'Addeo G, Cardinale D, Fiorilli R, Nappi F. A narrative review of diagnosis of infective endocarditis-imaging methods and comparison. Ann Transl Med 2020;8(23):1621. doi: 10.21037/atm-20-4555 after pacemaker implantation: infection rates and risk factors associated with infection in a population-based cohort study of 46299 consecutive patients. Eur Heart J 2011;32:991-8.

26. Sohail MR, Baddour LM. Role of PET Imaging in Management of Implantable Electronic Device Infection. JACC Cardiovasc Imaging 2016;9:291.

27. Sarrazin JF, Philippon F, Tessier M, et al. Usefulness of fluorine-18 positron emission tomography/computed tomography for identification of cardiovascular implantable electronic device infections. J Am Coll Cardiol 2012;59:1616-25.

28. Baddour LM, Wilson WR, Bayer AS, et al. Infective Endocarditis in Adults: Diagnosis, Antimicrobial Therapy, and Management of Complications. Circulation 2015;132:1435-86.

29. Mohananey D, Mohadjer A, Pettersson G, et al. Association of Vegetation Size With Embolic Risk in Patients With Infective Endocarditis: A Systematic Review and Meta-analysis. JAMA Intern Med 2018;178:502-10. 